\title{
sciendo
}

\section{PRENATAL PROGRAMMING OF THE SMALL INTESTINE IN PIGLETS: THE EFFECT OF SUPPLEMENTATION WITH 3-HYDROXY-3-METHYLBUTYRIC ACID (HMB) IN PREGNANT SOWS ON THE STRUCTURE OF JEJUNUM OF THEIR OFFSPRING*}

Ewa Tomaszewska ${ }^{1 \bullet}$, Łukasz Prost ${ }^{1}$, Piotr Dobrowolski², Deepesh K.P. Chand ${ }^{1}$, Janine Donaldson ${ }^{3}$, Anna Czech ${ }^{4}$, Renata Klebaniuk ${ }^{5}$, Julia Fabjanowska ${ }^{5}$, Siemowit Muszyński ${ }^{6}$

\author{
${ }^{1}$ Department of Animal Physiology, University of Life Sciences in Lublin, Akademicka 12, 20-950 Lublin, Poland \\ ${ }^{2}$ Department of Functional Anatomy and Cytobiology, Maria Curie-Sklodowska University, Akademicka 19, 20-033 Lublin, Poland \\ ${ }^{3}$ School of Physiology, Faculty of Health Sciences, University of the Witwatersrand, 7 York Road, Parktown, Johannesburg, 2193, South Africa \\ ${ }^{4}$ Department of Biochemistry and Toxicology, University of Life Sciences in Lublin, Akademicka 13, 20-950 Lublin, Poland \\ ${ }^{5}$ Institute of Animal Nutrition and Bromatology, University of Life Sciences in Lublin, Akademicka 13, 20-950 Lublin, Poland \\ ${ }^{6}$ Department of Biophysics, University of Life Sciences in Lublin, Akademicka 13, 20-950 Lublin, Poland \\ •Corresponding author: ewaRST@interia.pl
}

\begin{abstract}
When discussing the scale of the occurrence of diseases of the digestive system in farm animals, particularly pigs in the weaning period, it may be beneficial to study physiological and nutritional factors that could potentially affect the growth, development, and modelling of the structure and function of the digestive tract. Taking into account the reports on the beneficial effects of $\beta$-hydroxy-ß-methylbutyrate (HMB) administration in the prenatal period on the development of various systems it was assumed that the HMB supplementation to pregnant sows can influence intestinal development in the offspring during weaning. Thus, the present experiment was conducted to evaluate the effect of HMB treatment of pregnant sows on jejunum development in offspring at weaning. From 70th day until the 90th day of gestation, sows received either a basal diet $(\mathrm{n}=12)$ or the same diet supplemented with HMB $(\mathrm{n}=12)$ at the dose of $0.2 \mathrm{~g} / \mathrm{kg}$ of body weight/day. HMB given during prenatal time reduced the thickness of the longitudinal muscle; the apoptotic cell index in epithelium also significantly decreased after the HMB supplementation. Vasoactive intestinal (poly)peptide (VIP) expression in submucosal ganglia significantly increases in prenatally HMB treated piglets. The same strong reaction was observed with the expression of occludin, claudin-3, E-cadherin, and leptin in the jejunal epithelium. The obtained results indicate that the administration of HMB to pregnant sows significantly influenced the expression of VIP, leptin and some proteins of the intestinal barrier of their offspring less influencing the basal morphology.
\end{abstract}

Key words: offspring, pigs, pregnancy, weaning, HMB

Under the appropriate nutritional and environmental conditions in which the pregnant female is found, the genetic and phenotypic characteristics of the fetus are preserved in the prenatal period (So and $\mathrm{Ng}, 2005$ ). The influence of various endogenous and exogenous factors on the structural and functional development of organs and entire systems depends on the genetic sensitivity and the current period of fetal development as well as the status health of the pregnant female. As a result of programming, there is a permanent change in the regulation processes of the metabolic pathways (Matthews, 2001; Bertram and Hanson, 2002). As a result of programming, the metabolic pathways are permanently changed, and the process of adaptation to the new developmental conditions of the fetus must be as quick and efficient as pos- sible, so as not to disturb the entire development of the organism. The effects obtained as a result of the prenatal programming consist in accelerating or inhibiting the development, and somatic and hormonal maturity, which then determine the degree of independence in the postnatal period (Blicharski et al., 2017; Tomaszewska et al., $2020,2021)$. This is especially important at the time of weaning when there is once again a change in the feeding form. First, the fetus was nourished by the mother, then the newborn was fed with liquid food, and the next period like weaning the digestive system must adapt to the intake, digestion and utilization of nutrients from solid food. Therefore, the question of the extent to which the metabolic processes and the structure of the intestinal epithelium can be modulated to avoid disturbances in the no. WKF/S/32/2020. 
digestive system during weaning, which are especially observed in pig rearing, become important (Nowak et al., 2021).

In recent years, the effects of nutritional programming of various systems have been intensively studied. The nutritional factors can be toxic or they can improve the growth and development of the whole organism and individual systems, including digestive or skeletal systems (Tomaszewska et al., 2014; Blicharski et al., 2017; Duan et al., 2019; Tomaszewska et al., 2019, 2020; Dobrowolski et al., 2021; Tomczyk-Warunek et al., 2021). There is one study, which showed the important role of branched-chain amino acids in the biosynthesis of proteins that enable proper development and adaptation of the intestines in the postnatal period (Mou et al., 2019).

$ß$-hydroxy- $\beta$-methylbutyrate (HMB) is an endogenous metabolite of leucine, an essential amino acid, which shows anabolic and anticatabolic action, inhibits the breakdown of proteins and protects muscles from damage. It is produced naturally when leucine is oxidized in the cell cytoplasm, mainly in the liver and muscles (Nissen and Abumrad, 1997; Nissen et al., 2000; Lis et al., 2014). Administration of HMB to sows during pregnancy increases milk's fat content, the birth weight of piglets, and has a positive effect on the development of the skeletal system in the offspring (Flummer and Theil, 2012; Blicharski et al., 2017; Tomaszewska et al., 2018, 2019; Tomczyk-Warunek et al., 2021), increases the concentration of growth hormone and the activity of the bone alkaline phosphatase as a specific marker of bone formation processes in newborns, which in turn lead to the increase of the bone mass tissue (Tatara et al., 2007; Blicharski et al., 2017; Tomaszewska et al., 2019). Research results also show that administering HMB to lactating sows increases the energy and immunological value of milk and colostrum and increases the fat content of milk (Nissen et al., 1994).

The most common health problem in swine husbandry besides clostridial infection and coccidiosis is Escherichia coli (E. coli) infection called colibacillosis, a disease of the small intestine. It is a disease affecting both indoor and outdoor herds, can occur both chronically and sporadically. E. coli can have many clinical presentations in pigs of various age groups: diarrhea in suckling piglets, neurological signs in nursery pigs or urinary tract infections in sows. The known treatment of colibacillosis is the use of commonly available antibiotics (Francis, 1999). Thus, the prevalence of diseases of the digestive system and its dysfunction, especially during weaning, are associated with very high treatment costs and economic losses in animal husbandry (Xiong et al., 2019). The best way to prevent infection is providing a clean and dry environment for the piglets or sows` vaccination before farrowing. In the case of post-weaning diarrhoea, the prevention can be done by the supplementation of antimicrobials factors in the first nursery diet to neonatal piglets (Luppi, 2017). Nowadays, there is concern over the increased phenomenon of antimicrobial resistance among bacteria isolated from production animals. The risk linked to this phenomenon increases if antimicrobials (antibiotics or zinc oxide) are used inappropriately (Luppi, 2017). This is a challenge to find a new and effective way to prevent digestive system dysfunction. The scale of the occurrence of diseases of the digestive system in farm animals, including pigs in the weaning period, is an important need to study a set of physiological and nutritional factors that may potentially affect the growth, development, modelling of the structure and functioning of the digestive tract. However, there are no satisfactory data on the influence of HMB on the prenatal formation of the structure of the small intestine in pigs.

Taking into account the reports on the beneficial effects of HMB administration in the prenatal period on the development of various systems, including the skeletal system and striated skeletal muscles, it was decided to verify the hypothesis about the possibility of increasing the degree of intestinal development in the offspring during weaning by the HMB supplementation of pregnant sows.

To verify the above hypothesis, an experiment was carried out to evaluate the effect of prenatal administration of HMB in the diet of a pregnant sow on the postnatal development of the gastrointestinal tract in domestic pigs, with particular emphasis on the jejunum. In doing so, the present study examined (i) the integrity of intestinal barrier and basal morphology as well as the expression of selected hormones, and (ii) the effects of HMB supplementation. Thus, changes in immunoexpression and immunolocalization of vasoactive intestinal (poly) peptide and leptin were studied after HMB supplementation. Together, these results should provide additional fundamental information on the outcomes of HMB supplementation to pregnant sows, in relation to the small intestinal development of their offspring.

\section{Material and methods}

\section{Ethical approval}

All experimental procedures were approved by The Local Ethics Committee on Animal Experimentation of the University of Life Sciences in Lublin, Poland and complied with the Directive 2010/63/EU of the European Parliament and of the Council on the protection of animals used for scientific purposes. Throughout the experimental period, the health status of pregnant sows and piglets was regularly monitored by a veterinarian.

\section{Animals, breeding and experimental design}

The study was performed on 24 piglets born by 12 clinically healthy sows of the Polish Large White breed. Sows were sired by the same boar. Sows were singly housed in separated cages under standard rearing conditions (controlled temperature, humidity and 12:12-h light-dark cycle) with free access to fresh water and fed an amount of $3.0 \mathrm{~kg}$ of feed mixture per sow during late 
gestation with a balanced standard commercial diet for pregnant and lactating sows commonly used (Grela and Skomial, 2015). The feed mixture used for sows during pregnancy and lactation contained in $1 \mathrm{~kg}$ : $13.2 \mathrm{MJ} \mathrm{ME}$, $162 \mathrm{~g}$ of crude protein, $9.5 \mathrm{~g}$ of lysine and the scheme of the main essential amino acids proportion was lysine 100\%: methionine+cysteine 60\%: threonine 65\%: tryptophan $22 \%$. A two-phase feeding system for pregnant sows was introduced and the composition and amounts of feed were controlled in each phase of gestation.

Primiparous sows were used in the control and experimental groups to avoid the effect of the number of pregnancies on the birth weight in the offspring. The sows in both groups were ca. 10 months old and mated during the third oestrus.

To investigate the effects of prenatal treatment with a leucine metabolite on cartilage, $\beta$-hydroxy- $\beta$ methylbutyrate (HMB; Lonza, Basel, Switzerland) was administered to sows in mid-pregnancy. The sows were randomly assigned into two weight and age-matched groups (6 sows in each group). The sows were fed with a standard diet (control) and a diet supplemented with $\mathrm{HMB}(0.2 \mathrm{~g} / \mathrm{kg}$ of body weight/every day) in the morning meals from the 70th to 90th day of gestation. The dose of HMB was determined from a previous study, where we used HMB in a dose of $0.2 \mathrm{~g} / \mathrm{kg} /$ every day and a significant effect on enamel surface development of the offspring was found at this dose (Świetlicka et al., 2016; Tomaszewska et al., 2018). The gestation length did not differ between the control and HMB-treated sows.

All piglets born by physiological partum had no congenital changes. There were no stillbirths. Females and males used in the study were treated in the same manner. Piglets born by sows fed the control diet belonged to the control group of males $(n=6)$ and females $(n=6)$, and piglets born by HMB-treated sows belonged to the HMB group of males $(n=6)$ and females $(n=6)$. All offspring were held with their mothers without translocation between sows and fed naturally with colostrum and then with sow's milk, then at the age of 35 days, randomly chosen piglets were euthanized by intravenous injections of lethal doses of pentobarbitalum natrium (Morbital, Biowet, Puławy, Poland).

\section{Tissue collection and histomorphometry analysis}

Ten $\mathrm{mm}$ long segments of the jejunum $(50 \%$ of the total intestinal length of the jejunum) were taken from each piglet. Tissues were fixed in $4 \%$ buffered formaldehyde ( $\mathrm{pH} 7.0)$ for $24 \mathrm{~h}$, dehydrated in a graded series of ethanol, cleared with a nonpolar solvent, and then embedded in paraffin. $4 \mu \mathrm{m}$ thick cross-sections were cut with a microtome (Microm HM 360, Walldorf, Germany), then placed on one microscopic slide (Polysine ${ }^{\mathrm{TM}}$, Menzel Glaser, Braunschweig, Germany). For histomorphometry analysis, slides were stained with Masson's trichrome (Suvarna et al., 2013) and observed using a CX43 (Olympus, Tokyo, Japan) microscope. Collected microscopic images were examined blindly by an associate who was not aware of the treatment using graphical analysis software: Olympus cellSens (Olympus, Tokyo, Japan) and ImageJ (Schneider et al., 2012).

The following morphometric parameters were analyzed: the thickness of mucosa, submucosa, external muscular layer, and villar epithelium; enterocyte number; the number of villi, active and inactive crypts; villus length and thickness; crypt depth and width; area of the small intestine absorptive surface (Dobrowolski et al., 2021). For enteric ganglia (myenteric and submucosal), the following morphometric parameters were analyzed: the cross-section area, the perimeter, and circularity. The measurements of each variable were made on three separate tissue sections, on at least ten different areas of each section. The measurements were then averaged and expressed as the mean value of calculated parameters for each piglet.

\section{Immunohistochemistry}

Immunohistochemical staining was performed according to the protocols provided by the producer of the antibodies (Abcam, Cambridge, UK). Following primary antibodies were used: rabbit polyclonal anti-Ki67 antibody (ab15580); mouse monoclonal anti-neurofilament heavy (poly)peptide antibody to localize myenteric and submucosal ganglia (ab187374); mouse monoclonal antiactive caspase-3 (ab208161); rabbit polyclonal anti-VIP (ab22736), rabbit polyclonal anti-claudin-3 (ab15102), rabbit polyclonal anti-E-cadherin (ab15148), rabbit polyclonal anti-occludin (ab222691); rabbit polyclonal anti-leptin (ab16227, Abcam, Cambridge, UK, dilution 1:200), rabbit monoclonal anti-CD3 (ab16669). The immunoreaction was verified with the negative controls, treated by identical immunohistochemical staining, excluding the application of the primary antibody (HułasStasiak et al., 2020).

Microscopic images were subjected to further analysis. For Ki-67 and claudin-3, the proliferating and apoptotic cell indexes (the per cent of proliferating or apoptotic cells in relation to all the gland cells) were determined respectively. For neurofilament detection the cross-sectional area of the nerve ganglion; sphericity, perimeter, the minimal and mean diameter of the ganglion and mean Feret diameter (the distance between the two tangential lines restricting the object perpendicular to that direction) were determined using ImageJ software.

The intensity of immunoexpression was measured both by determining the percentage of cells with a positive response and by the quantitative comparison of mean pixel intensity in the photomicrographs, which were firstly converted into negatives and then into 8-bit grey-scale digital images, with a scale from 0 (white pixel) to 255 (black pixel), where the higher the pixel value, the higher the intensity of the immunohistochemical reaction. The intensity of the immunoreaction in each of the analyzed digital images was measured in 10 randomly selected areas of the positive signal. The analyses were done blindly 
by an associate who was not aware of the treatment using ImageJ software.

\section{Statistical analysis}

Data are presented as means \pm standard deviation. In all analyses, the single pig was considered as an experimental unit. A Shapiro-Wilk normality test was applied to test the normality of the data; the homogeneity of variances was assessed using Levene's test. Normally distributed variables were analyzed using a two-tailed Student's t-test or t-test with Welch's correction when normally distributed data lack equal variances; non-parametric data were analyzed using a Mann-Whitney U test. For all tests, a P-value $<0.05$ was established as statistically significant. All statistical procedures were performed using Statistica 13.0 (Tibco, Palo Alto, CA, USA) software.

\section{Results}

\section{Jejunal morphology}

No macroscopic changes indicating an anastomotic failure or infection were found during dissection. The analysis of basal morphology of the jejunal wall showed that HMB given during prenatal time reduced the thickness of the outer layer of muscularis, which led to the reduction of the total thickness of muscularis in piglets. The number of apoptotic cells also significantly decreased after the HMB supplementation (Table 1). Other changes were not observed. The analysis of basal morphology of jejunal villi showed the lack of the effect of the prenatal HMB supplementation in piglets (Table 2). Taking into account the basal morphology of piglets' jejunal crypts, there was observed a decrease of the depth after the prenatal HMB supplementation (Table 3). The size and geometry of both ganglia were unchanged (Table 4).

\section{VIP}

Immunolocalization and immunoexpression of

Immunolocalisation of vasoactive intestinal (poly) peptide (VIP) showed that VIP immune reactive cells were observed in both the myenteric and submucosal ganglia in the control and HMB groups. The intensity of immune reaction was significantly higher in myenteric ganglia in the control group compared with the HMB group, while VIP expression in submucosal ganglia was very strong in prenatally HMB treated piglets compared with the control piglets (Figure 1).

Table 1. The effect of HMB on basal morphology of intestinal wall of piglets at the age of 35 days

\begin{tabular}{|c|c|c|c|}
\hline Item & Control & HMB & P-value \\
\hline Mucosa thickness $(\mu \mathrm{m})$ & $593 \pm 122$ & $565 \pm 96$ & n.s. \\
\hline Submucosa thickness $(\mu \mathrm{m})$ & $38.3 \pm 12.2$ & $39.0 \pm 16.3$ & n.s. \\
\hline Thickness of inner muscle layer $(\mu \mathrm{m})$ & $57.0 \pm 11.2$ & $54.5 \pm 8.5$ & n.s. \\
\hline Thickness of outer muscle layer $(\mu \mathrm{m})$ & $47.2 \pm 8.6$ & $26.0 \pm 8.1$ & $* * *$ \\
\hline Total thickness of muscularis $(\mu \mathrm{m})$ & $106.6 \pm 9.12$ & $79.7 \pm 9.5$ & $* * *$ \\
\hline Muscle to mucosa thickness ratio & $0.18 \pm 0.08$ & $0.14 \pm 0.09$ & n.s. \\
\hline Absorption surface $\left(\mathrm{mm}^{2}\right)$ & $6.17 \pm 1.49$ & $6.58 \pm 2.23$ & n.s. \\
\hline Apoptotic cells index $(\%)$ & $11.79 \pm 2.21$ & $9.32 \pm 1.97$ & $* *$ \\
\hline Ki index $(\%)$ & $52.7 \pm 15.6$ & $54.8 \pm 16.8$ & n.s. \\
\hline CD3 number $\left(/ \mathrm{mm}^{2}\right)$ & $874 \pm 368$ & $537 \pm 295$ & n.s. \\
\hline
\end{tabular}

Data are presented as mean \pm standard deviation $(\mathrm{n}=12)$. Significance was established using a two-tailed Student's t-test (normally distributed data), Welch's test (normally distributed data with unequal variances) or Mann-Whitney test (for pairwise comparisons with at least one non-normally distributed dataset); n.s. - not significant; **P $<0.01 ; * * * \mathrm{P}<0.001$.

Table 2. The effect of HMB on basal morphology of intestinal villus of piglets at the age of 35 days

\begin{tabular}{|c|c|c|c|}
\hline Item & Control & HMB & P-value \\
\hline Villi length $(\mu \mathrm{m})$ & $304 \pm 68$ & $302 \pm 95$ & n.s. \\
\hline Villi width $(\mu \mathrm{m})$ & $88.8 \pm 21.1$ & $98.0 \pm 19.7$ & n.s. \\
\hline Total number of villi/mm & $14.3 \pm 2.08$ & $7.27 \pm 1.85$ & n.s. \\
\hline Number of undamaged villi/mm & $1.73 \pm 1.07$ & $0.81 \pm 1.17$ & n.s. \\
\hline Number of damaged villi/mm & $6.39 \pm 1.91$ & $6.43 \pm 4.15$ & n.s. \\
\hline Enterocytes thickness $(\mu \mathrm{m})$ & $26.8 \pm 4.2$ & $24.5 \pm 3.5$ & n.s. \\
\hline Villus/crypt ratio & $2.53 \pm 1.21$ & $2.22 \pm 1.23$ & n.s. \\
\hline Enterocytes number $/ 100 \mu \mathrm{m}$ & $16.9 \pm 4.7$ & $22.4 \pm 9.7$ & n.s. \\
\hline Number of goblet cells/100 $\mu \mathrm{m}$ & $0.93 \pm 1.12$ & $1.44 \pm 1.41$ & n.s. \\
\hline Enterocytes to goblet cells ratio & $18.21 \pm 4.20$ & $15.17 \pm 6.57$ & n.s. \\
\hline
\end{tabular}

Data are presented as mean \pm standard deviation $(n=12)$. Significance was established using a two-tailed Student's t-test (normally distributed data), Welch's test (normally distributed data with unequal variances) or Mann-Whitney test (for pairwise comparisons with at least one non-normally distributed dataset); n.s. - not significant. 
Table 3. The effect of HMB on basal morphology of intestinal crypts of piglets at the age of 35 days

\begin{tabular}{|c|c|c|c|}
\hline Item & Control & HMB & P-value \\
\hline Crypts depth $(\mu \mathrm{m})$ & $120 \pm 15.6$ & $93.5 \pm 13.8$ & $* * *$ \\
\hline Crypts width $(\mu \mathrm{m})$ & $33.3 \pm 13.7$ & $44.6 \pm 15.9$ & n.s. \\
\hline Total number of crypts/mm & $15.9 \pm 3.5$ & $15.8 \pm 3.6$ & n.s. \\
\hline Number of open crypts/mm & $1.91 \pm 1.81$ & $1.55 \pm 1.60$ & n.s. \\
\hline Number of closed crypts/mm & $13.6 \pm 3.7$ & $14.7 \pm 3.9$ & n.s. \\
\hline
\end{tabular}

Data are presented as mean \pm standard deviation $(\mathrm{n}=12)$. Significance was established using a two-tailed Student's t-test (normally distributed data), Welch's test (normally distributed data with unequal variances) or Mann-Whitney test (for pairwise comparisons with at least one non-normally distributed dataset); n.s. - not significant; $* * * \mathrm{P}<0.001$.

Table 4. The effect of HMB on basal morphology of intestinal ganglia of piglets at the age of 35 days

\begin{tabular}{|c|c|c|c|}
\hline Item & Control & HMB & $\mathrm{P}$-value \\
\hline \multicolumn{4}{|l|}{ Myenteric ganglion } \\
\hline The cross-section area $\left(\mu \mathrm{m}^{2}\right)$ & $1624 \pm 1385$ & $906 \pm 639$ & n.s. \\
\hline Perimeter $(\mu \mathrm{m})$ & $196 \pm 123$ & $161 \pm 81$ & n.s. \\
\hline Circularity & $0.22 \pm 0.17$ & $0.25 \pm 0.24$ & n.s. \\
\hline \multicolumn{4}{|l|}{ Submucosal ganglion } \\
\hline The cross-section area $\left(\mu \mathrm{m}^{2}\right)$ & $988 \pm 622$ & $928 \pm 815$ & n.s. \\
\hline Perimeter $(\mu \mathrm{m})$ & $162 \pm 77$ & $149 \pm 85$ & n.s. \\
\hline Circularity & $0.21 \pm 0.25$ & $0.24 \pm 0.22$ & n.s. \\
\hline
\end{tabular}

Data are presented as mean \pm standard deviation $(\mathrm{n}=12)$. Significance was established using a two-tailed Student's t-test (normally distributed data), Welch's test (normally distributed data with unequal variances) or Mann-Whitney test (for pairwise comparisons with at least one non-normally distributed dataset); n.s. - not significant.

\section{Immunolocalization and immunoexpression of proteins of intestinal barrier}

The expression of occludin, an integral plasmamembrane protein located at the tight junctions, was of lower intensity in the control group, irrespective it was analyzed across the whole epithelium or including separately crypts and villi. The analysis across the epithelium showed that the intensity of the immune reaction was stronger in crypts comparing with villi in the control group. It was not observed in the HMB group, where the occluding intensity was similar in both parts analyzed. Moreover, a stronger intensity of the occludin expression was generally in the HMB group irrespective of the part analyzed (Figure 2).

The expression of claudin-3, another integral plasma-membrane protein located at the tight junctions and serving as a physical barrier to prevent solutes and water from passing freely through the paracellular space, showed that claudin-3 was present in both groups along the whole epithelium, but the significantly stronger reaction was in the HMB group comparing with the control group irrespective of the part analyzed (Figure 3 ).
The expression of E-cadherin, a type of cell adhesion molecule (CAM) that are important in the formation of adherens junctions to bind cells with each other, showed that E-cadherin also was present along the whole epithelium in both groups of piglets. Taking into account the control group, the reaction was stronger in crypts comparing with villi, while in the HMB group the reaction was similar in both parts of the epithelium. Further, immunoexpression of E-cadherin was significantly stronger, irrespective of the part analyzed in the HMB group than in the control group (Figure 4).

The expression of leptin, a hormone involved in the regulation of energy balance through the inhibition of hunger, is presented in the jejunum in the control and HMB groups of piglets in Figure 5. A stronger immune reaction of leptin was observed in the crypts, epithelium villi and both ganglia of enteric nervous system in the HMB group. Taking into account the whole epithelium, this reaction also was stronger in the HMB group comparing to the control group. 


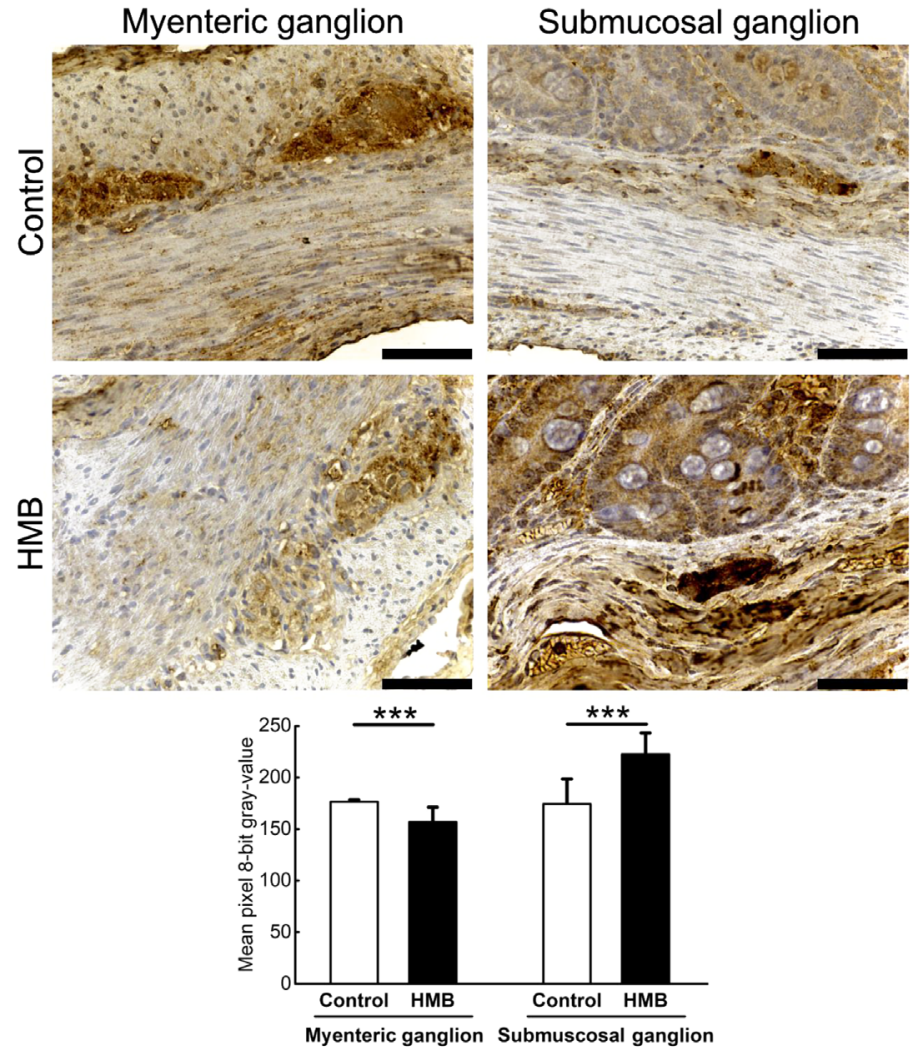

Figure 1. Representative photomicrographs of the immunohistochemical reactions for vasoactive intestinal (poly)peptide (VIP) in the myenteric and submucosal ganglia in jejunum of control and prenatally HMB-exposed pigs. All the scale bars represent $40 \mathrm{um}$. Bar graphs show the intensity of expression of VIP in ganglia measured by the comparison of the pixel brightness value in the microscopic images converted to 8-bit grayscale - the higher the pixel value, the higher the intensity of the immunohistochemical reaction. Graph shows mean \pm standard deviation. Significance was established using a two-tailed Student's t-test (normally distributed data), Welch's test (normally distributed data with unequal variances) or Mann-Whitney test (for pairwise comparisons with at least one non-normally distributed dataset); $* * * \mathrm{P}<0.001$
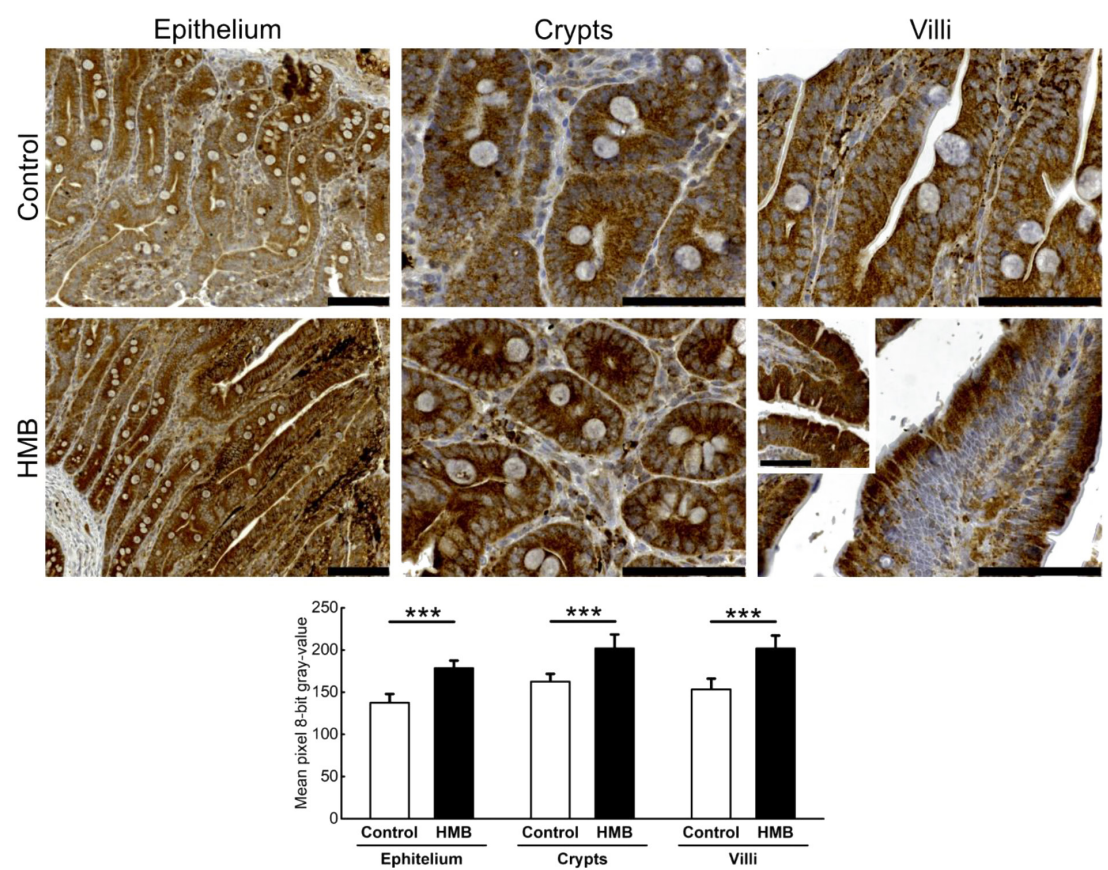

Figure 2. Representative photomicrographs of the immunohistochemical reactions for occludin in the epithelium, crypts and villi in jejunum of control and prenatally HMB-exposed pigs. All the scale bars represent $100 \mathrm{um}$. Bar graphs show the intensity of expression of occludin in intestinal areas measured by the comparison of the pixel brightness value in the microscopic images converted to 8-bit grayscale - the higher the pixel value, the higher the intensity of the immunohistochemical reaction. Graph shows mean \pm standard deviation. Significance was established using a two-tailed Student's t-test (normally distributed data), Welch's test (normally distributed data with unequal variances) or Mann-Whitney test (for pairwise comparisons with at least one non-normally distributed dataset); ${ }^{* * *} \mathrm{P}<0.001$ 

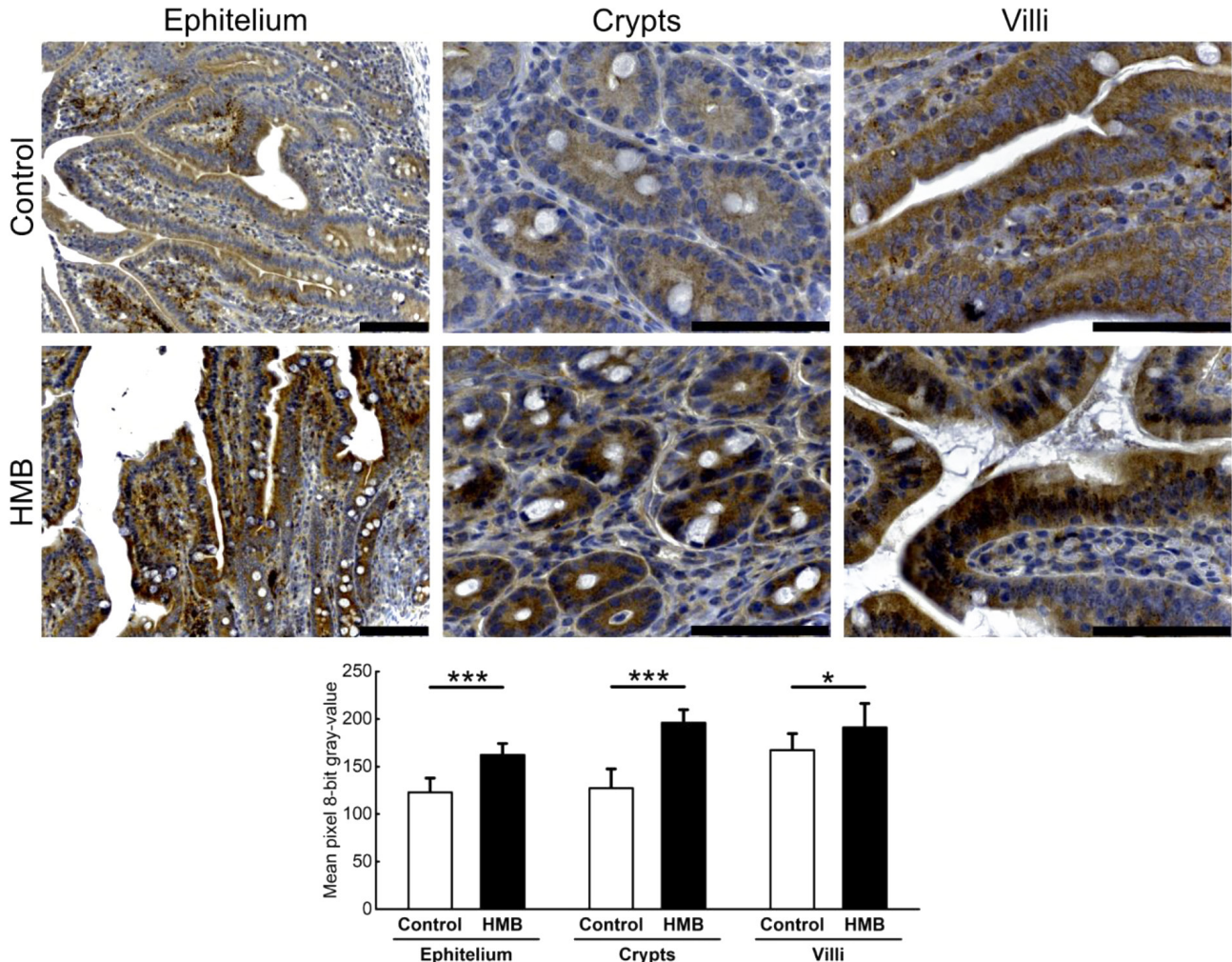

Figure 3. Representative photomicrographs of the immunohistochemical reactions for claudin-3 in the epithelium, crypts and villi in jejunum of control and prenatally HMB-exposed pigs. All the scale bars represent $100 \mathrm{um}$. Bar graphs show the intensity of expression of claudin-3 in intestinal areas measured by the comparison of the pixel brightness value in the microscopic images converted to 8-bit grayscale - the higher the pixel value, the higher the intensity of the immunohistochemical reaction. Graph shows mean \pm standard deviation. Significance was established using a two-tailed Student's t-test (normally distributed data), Welch's test (normally distributed data with unequal variances) or Mann-Whitney test (for pairwise comparisons with at least one non-normally distributed dataset); $* \mathrm{P}<0.01 ; * * * \mathrm{P}<0.001$
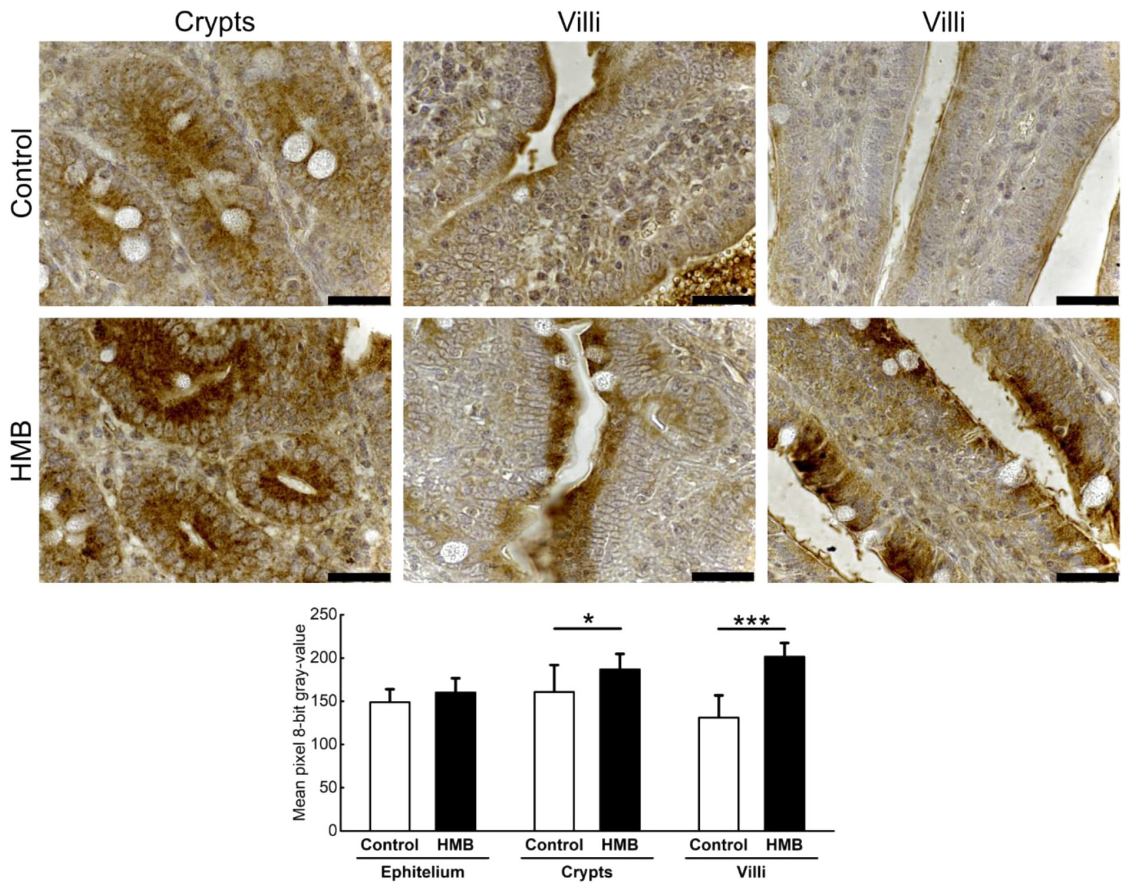

Figure 4. Representative photomicrographs of the immunohistochemical reactions for E-cadherin in the crypts and villi in jejunum of control and prenatally HMB-exposed pigs. All the scale bars represent $100 \mathrm{um}$. Bar graphs show the intensity of expression of E-cadherin in the jejunal epithelium, crypts and villi measured by the comparison of the pixel brightness value in the microscopic images converted to 8-bit grayscale the higher the pixel value, the higher the intensity of the immunohistochemical reaction. Graph shows mean \pm standard deviation. Significance was established using a two-tailed Student's t-test (normally distributed data), Welch's test (normally distributed data with unequal variances) or Mann-Whitney test (for pairwise comparisons with at least one non-normally distributed dataset); ${ }^{*} \mathrm{P}<0.01 ; * * * \mathrm{P}<0.001$ 




Figure 5. Representative photomicrographs of the immunohistochemical reactions for leptin in the ganglia, crypts and villi in jejunum of control and prenatally HMB-exposed pigs. White arrow shows submucosal ganglion and black arrow shows myenteric ganglion. All the scale bars represent $100 \mathrm{um}$. Bar graphs show the intensity of expression of leptin in the jejunal epithelium, crypts and villi measured by the comparison of the pixel brightness value in the microscopic images converted to 8-bit grayscale - the higher the pixel value, the higher the intensity of the immunohistochemical reaction. Graph shows mean \pm standard deviation. Significance was established using a two-tailed Student's t-test (normally distributed data), Welch's test (normally distributed data with unequal variances) or Mann-Whitney test (for pairwise comparisons with at least one non-normally distributed dataset); ${ }^{* * *} \mathrm{P}<0.001$

\section{Discussion}

The postnatal development of all organisms depends primarily on genes. However, as it was shown by studies conducted on various species of animals, the postnatal development of an organism is also determined in the prenatal time by non-genetic factors (Hales et al., 1991; Blicharski et al., 2017; Tomaszewska et al., 2018). Important factors that largely determine the proper prenatal development are the nutrients present in the diet of a pregnant female (Czech et al., 2020). Nutrients can be different, they can be basic nutrients playing a building, energy or regulatory role, and non-nutrients, such as toxic substances (Tomaszewska et al., 2014, 2020; Rudyk et al., 2020).

Supplements are among the nutrients that can be found in a pregnant female's diet. However, knowledge about the effects of most supplements on the nutrition of pregnant females is incomplete (Tomaszewska et al., 2018; Milart et al., 2019). The action of all these factors may have positive effects or inhibit the overall development of offspring's organism. This effect is observed not only with body weight and bone tissue but also in the digestive system, which largely determines the survival rate, animal health and production effects. All processes related to food intake, digestion and grinding, utilization and removal of undigested remains dependent on the degree of development of the digestive system in the prenatal period. The digestive tract is also the first place where an individual comes into contact with allergens and pathogens. Therefore, it should be remembered that the functional and structural preparation of the gastrointestinal tract to perform all these functions takes place already in the prenatal period. Mou et al. (2019) presented the role of branched amino acids not only in general growth but also in proteosynthesis of the visceral tissue of the internal organs, possibly due to the holistic growth of the organism exposed to $\mathrm{HMB} /$ leucine.

The current study was focused on the structural adaptation of the small intestine in offspring from sows supplemented with HMB. To our knowledge, there are no studies on pregnant sows supplemented with HMB with regards to the postnatal development of the gastrointestinal tract in pigs. For this reason, it is difficult to directly 
compare and discuss the obtained results in the context of the results described in previously conducted studies. However, there are a few studies that show that HMB has the potential in ameliorating acute intestinal toxicity and gastrointestinal injury induced by radiation (Yavas et al., 2013; Zheng et al., 2020). Zheng et al. (2020) has shown that HMB supplementation significantly increased jejunal villus height and in turn elevated the villus height-tocrypt depth ratio.

Our study showed very few changes in the basal jejunal morphology including the reduction of myenteron thickness. The intestinal myenteron participates in the mixing of chyme and moving it along the digestive tract. Based on obtained results it could be assumed that these changes will lead to the weakening or strengthening of bowel movements and following slowing or acceleration of the chyme passage; and what is influence on generating myoelectric potential. There is another important issue, which should be further investigated. It should be considered whether the smooth muscle is as susceptible to differentiation and growth by activating the mTORC1 pathway as skeletal muscle. The lack of other changes can suggest that the function of the intestine was unaffected by the prenatal HMB supplementation. This means that both the mucus layer as a part of the mucosal barrier and the mucosal barrier by itself remains intact (no change in the number of goblet cells), but it does not indicate that they are structures that cannot be programmed. Acrylamide significantly disrupts both the intestinal barrier and the structure of the small intestine in newborn guinea pigs as well as HMB in minks when animals are exposed to it during the prenatal time (Tomaszewska et al., 2014; Dobrowolski et al., 2021).

The lack of changes in the structures of the intestine, in the structure of the villi and crypts, proves (including the proliferation index) that HMB administered to a pregnant female did not affect the processes of renewal and regeneration of the intestinal epithelium or the shape of the villi. The number and shape of the villi largely determine the surface of the intestinal mucosa (in the presented piglets there is no change in the absorption surface), which in turn determines the processes of digestion and absorption. Changing the shape of the villi, e.g. from finger-like shape to leafy, can already cause a significant reduction in the surface of the intestinal mucosa. The epithelium that covers the intestinal mucosa is constantly renewed and regenerated. The site of renewal is the intestinal crypts (no changes in the jejunum in piglets after $\mathrm{HMB}$ ), from which cells, as a result of divisions, move along the lateral surface of the villi towards the top of the villi, where they are exfoliated. Maintaining the correct shape and size of the villi depends on the dynamic balance between the renewal and exfoliation processes, which was not probably disturbed in piglets prenatally exposed to HMB. Therefore, it should be assumed that HMB supplementation did not disturb the proliferation and exfoliation in any way, nor did it intensify or inhibit the mentioned processes. The observed lack of influ- ence is a very good effect because the renewal processes are very intense and the action of any factor may cause a change in the rate of any of the described processes and affect the shape and size of the intestinal villi. For example, the lack of nutrients inhibits exfoliation, and on the other hand, increased exfoliation, even when the processes of cell proliferation and migration are too intense, which may turn out to be insufficient for reconstruction, can lead to villi atrophy. Moreover, the current study showed a decrease in the apoptosis process, which is also described in skeletal muscles (Hao et al., 2011).

Administration of HMB to pregnant sows also had a positive effect on the small intestine barrier. The gutepithelial barrier consists of a complex system of proteinprotein networks linking the contiguous cell and closing the intercellular space. These zones are impermeable to macromolecular substances and are generally more permeable in the small intestine compared to the rest of the intestine. The performed immunohistochemical reactions towards the expression of claudin-3, the tight junction zone protein as well as the expression of E-cadherin, the adhesion zone protein of the adherens junction, show that the endothelial cells of the piglet's mucosa show greater coherence, leading to an increase in the tight junction of the intestinal barrier.

The impermeability of the intestinal epithelium due to the presence of closing edges or adhesion between enterocytes together with the mucous barrier is one of the elements of the non-cellular non-specific immune response. Improving the structure of the intestinal barrier may reduce inflammation or cancer processes later in life when the living organism is in particularly unfavourable environmental conditions with a changed nutritional profile (weaning or changing the diet or environment). Absorption in the small intestine occurs in two ways. One route is through transcellular uptake through enterocyte structures. The second route is intracellular absorption through proteins that connect adjacent intestinal epithelial cells. The damage of the intestinal epithelium and the mucosa barrier involved the presence of zonulin-dependent changes in gut-epithelial and gut-vascular barriers and makes it possible for bacteria and bacterial toxins to enter, and the depletion of the intestinal bacterial flora increases the susceptibility of the mucosa to pathogens, their metabolites and other inflammatory signals, leading to intestinal dysbiosis and reduction of positive local and general immune response (Groschwitz and Hogan, 2009).

Another important effect observed in the current study is the change of the VIP expression in the enteric nervous system after the HMB maternal supplementation. VIP is extensively distributed in central and peripheral tissues, where it acts as a neurotransmitter and neuromodulator. VIP has several actions in the periphery, including vascular and non-vascular smooth muscle relaxation, vasodilatation and electrolyte secretion. It stimulates the secretion of water, bicarbonate, bile, pepsinogen; and has also been shown to possess both anti-inflammatory and 
anti-apoptotic properties (Gonzalez-Rey and Delgado, 2005).

Thus, from the point of view of that anti-inflammatory VIP property, its potential role in the pathogenesis of gastrointestinal diseases cannot be underestimated (Delgado et al., 2004). Additionally, it is suggested that VIP has antimicrobial, antibacterial, and antifungal properties. VIP displayed antimicrobial activity against Streptococcus mutans, Lactobacillus acidophilus, Enterococcus faecalis, Escherichia coli, Pseudomonas aeruginosa and Candida albicans (El Karim et al., 2008).

VIP affects blood vessels, suppresses gastric acid secretion, stimulates the secretion of pancreatic and intestinal juices, water and chlorine ions in the intestines (reducing their absorption), relaxes the smooth muscle of the gastrointestinal tract, induces glycogenolysis and gluconeogenesis in hepatocytes, and also affects immunity (Said, 1991; Bik, 2007; Winzell and Ahrén, 2007). In addition, studies conducted on mice prove that VIP is involved in the regulation of appetite and satiety, and plays a key role in regulating body weight, influencing its increase (Vu et al., 2015).

The current study showed that HMB especially increased the VIP expression in myenteric ganglia. It was a possible compensatory mechanism counteracting the dramatic decrease of brush border enzyme activity and electrolytes secretion in the small intestine at the weaning in pigs. Its higher presence with the connection of improved intestinal barrier and anti-inflammatory and anti-bacterial properties after the HMB supplementation can help to keep a healthy gut (Xiong et al., 2019). Moreover, other studies on several animals have showed that VIP expression is changed by the inflammatory process, which correlates with altered smooth muscle contractility (Arciszewski et al., 2009).

The next important finding of the current study was linked with the increase of the expression of leptin in the intestinal epithelium. On the other hand, the increase in its concentration in the peripheral blood was not observed. Leptin increases the insulin sensitivity of peripheral tissues, which increases the glucose reuptake (Friedman, 2014). Moreover, leptin is produced physiologically by the intestinal epithelium where plays a role of growth factor for the epithelium in the digestive tract (Pearson et al., 2001). Leptin is also a hormone that is involved in the functioning of the gut-brain axis and affects the body's energy expenditure (Park and Ahima, 2014).

It seems that the HMB use as a factor protecting against health problems at weaning in swine production could be a good strategy, which can be introduced to breeding. A recent study shows that after maternal HMB supplementation piglets are heavier at birth and weaning and slaughter (Tatara et al., 2007; Blicharski et al., 2017). That form of administration reducing the need for piglet supplementation in favour of supplementation of the pregnant sow is extremely important from the point of view of animal welfare.
The obtained results indicate that the administration of HMB to pregnant sows significantly influences the postnatal development of the small intestine of their offspring, but as it can be seen from the presented experience, it is difficult to clearly explain the phenomenon of prenatal programming of the gastrointestinal function at this stage. Further studies should be performed to elucidate the observed changes in the piglets' digestive tract.

\section{Conflict of Interests Statement}

The authors declare that they have no conflict of interest regarding the publication of this article.

\section{References}

Arciszewski M.B., Nowakowski Z., Wasowicz K., Całka J. (2009). Expression of vasoactive intestinal polypeptide, substance $\mathrm{P}$ and neuropeptide $\mathrm{Y}$ in jejunal enteric nerves is altered in rabbits suffering from long term Trichinella spiralis infection: an immunohistochemical study. Vet. Med., 54: 589597.

Bertram C.E., Hanson M.A. (2002). Prenatal programming of postnatal endocrine responses by glucocorticoids. Reproduction, 124: 459467.

Bik W. (2007). Vasoactive intestinal peptide-immunomodulatory factor and its role in respiratory diseases (in Polish). Post. Nauk Med., 10: 408413.

Blicharski T., Tomaszewska E., Dobrowolski P., Hułas-Stasiak M., Muszyński S. (2017). A metabolite of leucine ( $\beta$-hydroxy- $\beta$ methylbutyrate) given to sows during pregnancy alters bone development of their newborn offspring by hormonal modulation. PLoS One, 12: e0179693.

Czech A., Grela R.E., Kiesz M., Kłys S. (2020). Biochemical and haematological blood parameters of sows and piglets fed a diet with a dried fermented rapeseed meal. Ann. Anim. Sci., 20: 535-550.

Delgado M., Pozo D., Ganea D. (2004). The significance of vasoactive intestinal peptide in immunomodulation. Pharmacol. Rev., 56: 249290.

Dobrowolski P., Muszyński S., Donaldson J., Jakubczak A., Żmuda A., Taszkun I., Rycerz K., Mielnik-Błaszczak M., Kuc D., Tomaszewska E. (2021). The effects of prenatal supplementation with $\beta$-hydroxy- $\beta$-methylbutyrate and/or alpha-ketoglutaric acid on the development and maturation of mink intestines are dependent on the number of pregnancies and the sex of the offspring. Animals, 11: 1468.

Duan Y., Li F., Song B., Zheng C., Zhong Y., Xu K., Kong X., Yin Y., Wang W., Shu G. (2019). $\beta$-hydroxy- $\beta$-methyl butyrate, but not $\alpha$-ketoisocaproate and excess leucine, stimulates skeletal muscle protein metabolism in growing pigs fed low protein diets. J. Funct. Foods, 52: 34-42.

El Karim I.A., Linden G.J., Orr D.F., Lundy F.T. (2008). Antimicrobial activity of neuropeptides against a range of micro-organisms from skin, oral, respiratory and gastrointestinal tract sites. J. Neuroimmunol., 200: 1116.

Flummer C., Theil P.K. (2012). Effect of $\beta$-hydroxy $\beta$-methyl butyrate supplementation of sows in late gestation and lactation on sow production of colostrum and milk and piglet performance. J. Anim. Sci., 90: 372-374.

Francis D.H. (1999). Colibacillosis in pigs and its diagnosis. Swine Health Prod., 7: 241244.

Friedman J. (2014). 20 years of leptin: leptin at 20: an overview. J. Endocrinol., 223: T1T8.

Gonzalez-Rey E., Delgado M. (2005). Role of vasoactive intestinal peptide in inflammation and autoimmunity. Curr. Opin. Investig. Drugs., 6: 11161123.

Grela E.R., Skomiał J. (2015). Nutritional recommendations and nutritional value of feed for pigs, 2nd ed. Institute of Physiology 
and Animal Nutrition of Polish Academy of Science, Jabłonna, Poland, pp. 195.

Groschwitz K.R., Hogan S.P. (2009). Intestinal barrier function: molecular regulation and disease pathogenesis. J. Allergy Clin. Immunol., 124: 320.

Hales C.N., Barker D.J., Clark P.M., Cox L.J., Fall C., Osmond C., Winter P.D. (1991). Fetal and infant growth and impaired glucose tolerance at age 64. Br. Med. J., 303: 10191022.

Hao Y., Jackson J.R., Wang Y., Edens N., Pereira S.L., Alway S.E. (2011). $\beta$-Hydroxy- $\beta$-methylbutyrate reduces myonuclear apoptosis during recovery from hind limb suspension-induced muscle fiber atrophy in aged rats. Am. J. Physiol. Regul. Integr. Comp. Physiol., 301: R701R715.

Hułas-Stasiak M., Jakubowicz-Gil J., Dobrowolski P., Grzesiak M., Muszyński S., Świątkiewicz M., Tomaszewska E. (2020). Regulation of folliculogenesis by growth factors in piglet ovary exposed prenatally to $\beta$-hydroxy- $\beta$-methylbutyrate (HMB). Ann. Anim. Sci., 20: 899-917.

Lis I., Bogdański P., Karolkiewicz J. (2014). The effect of $\beta$-hydroxy$\beta$-methylbutyrate (HMB) on muscle protein metabolism. Farm Współ., 7: 3241.

Luppi A. (2017). Swine enteric colibacillosis: diagnosis, therapy and antimicrobial resistance. Porc. Health Manag., 3: 16.

Matthews S.G. (2001). Antenatal glucocorticoids and the development brain: mechanisms of action. Semin Neonatol., 6: 309317.

Milart P., Paluszkiewicz P., Dobrowolski P., Tomaszewska E., Smolińska K., Dębińska I., Gaweł K., Walczak K., Bednarski J., Turska M., Raban M., Kocki T., Turski W.A. (2019). Kynurenic acid as the neglected ingredient of commercial baby formulas. Sci. Rep., 9: 6108.

Mou Q., Yang H-S., Yin Y-L., Huang P-F. (2019). Amino acids influencing intestinal development and health of the piglets. Animals, 9: 302 .

Nissen S., Abumrad N.N. (1997). Nutritional role of the leucine metabolite $\beta$-hydroxy- $\beta$-methylbutyrate (HMB). J. Nutr. Biochem., 8: 300311 .

Nissen S., Faidley T.D., Zimmerman D.R., Izard R., Fisher C.T. (1994). Colostral milk fat percentage and pig performance are enhanced by feeding the leucine metabolite beta-hydroxy-betamethyl butyrate to sows. J. Anim. Sci., 72: 2331-2337.

Nissen S., Sharp R.L., Panton L., Vukovich M., Trappe S., Fuller J.C. Jr. (2000). Beta-hydroxy-methylbutyrate (HMB) supplementation in humans is safe and decrease cardiovascular risk factors. J. Nutr., 130: 19371945.

Nowak P., Zaworska-Zakrzewska A., Frankiewicz A., KasprowiczPotocka M. (2021). The effects and mechanisms of acids on the health of piglets and weaners - a review. Ann. Anim. Sci., 21: 433-455.

Park H.K., Ahima R.S. (2014). Leptin signaling. F1000Prime Rep., 6: 73.

Pearson P.Y., O'Connor D.M., Schwartz M.Z. (2001). Novel effect of leptin on small intestine adaptation. J. Surg. Res., 97: 192-195.

Rudyk H., Tomaszewska E., Arciszewski M.B., Muszyński S., Tomczyk-Warunek A., Dobrowolski P., Donaldson J., Brezvyn O., Kotsyumbas I. (2020). Histomorphometrical changes in intestine structure and innervation following experimental fumonisins intoxication in male Wistar rats. Pol. J. Vet. Sci., 23: 7788.

Said S.I. (1991). Vasoactive intestinal polypeptide: biologic role in health and disease. Trends Endocrinol Metab., 2: 107-112.

Schneider C.A., Rasband W.S., Eliceiri K.W. (2012). NIH Image to ImageJ: 25 years of image analysis. Nat. Methods, 9: 671675.

So K-W., Ng P-C. (2005). Treatment and prevention of neonatal osteopenia. Curr. Pediatr., 15: 106-113.
Suvarna S.K., Layton C., Bancroft J.D. (2013). Bancroft's theory and practice of histological techniques, 7th ed. Churchill Livingstone, New York, NY, USA.

Świetlicka I., Muszyński S., Tomaszewska E., Dobrowolski P., Kwaśniewska A., Świetlicki M., Skic A., Gołacki K. (2016). Prenatally administered HMB modifies the enamel surface roughness in spiny mice offspring: An atomic force microscopy study. Arch. Oral Biol., 70: 2431.

Tatara M.R., Śliwa E., Krupski W. (2007). Prenatal programming of skeletal development in the offspring: effects of maternal treatment with $\beta$-hydroxy- $\beta$-methylbutyrate (HMB) on femur properties in pigs at slaughter age. Bone, 40: 1615-1622.

Tomaszewska E., Dobrowolski P., Puzio I., Prost Ł., Kurlak P., Sawczuk P., Badzian B., Hułas-Stasiak M., Kostro K. (2014). Acrylamide-induced prenatal programming intestine structure in guinea pig. J. Physiol. Pharmacol., 65: 107-115.

Tomaszewska E., Dobrowolski P., Świetlicka I., Muszyński S., Kostro K., Jakubczak A., Taszkun I., Żmuda A., Rycerz, K., Blicharski T., Jaworska-Adamu J. (2018). Effects of maternal treatment with $\beta$-hydroxy- $\beta$-metylbutyrate and 2-oxoglutaric acid on femur development in offspring of minks of the standard dark brown type. J. Anim. Physiol. Anim. Nutr., 102: e299-e308.

Tomaszewska E., Muszyński S., Dobrowolski P., Wiącek D., Tomczyk-Warunek A., Świetlicka I., Pierzynowski G.P. (2019). Maternal HMB treatment affects bone and hyaline cartilage development in their weaned piglets via the leptin/osteoprotegerin system. J. Anim. Physiol. Anim. Nutr., 103: 626-643.

Tomaszewska E., Dobrowolski P., Puzio I., Donaldson J., Muszyński S. (2020). Acrylamide-induced prenatal programming of bone structure in mammal model. Ann. Anim. Sci., 20: 1257-1287.

Tomaszewska E., Burmańczuk N., Dobrowolski P., Świątkiewicz M., Donaldson J., Burmańczuk A., Mielnik-Błaszczak M., Kuc D., Milewski S., Muszyński S. (2021). The protective role of alphaketoglutaric acid on the growth and bone development of experimentally induced perinatal growth-retarded piglets. Animals, 11: 137.

Tomczyk-Warunek A., Blicharski T., Jarecki J., Dobrowolski P., Muszyński S., Tomaszewska E., Rovati L.C. (2021). The effect of maternal HMB supplementation on bone mechanical and geometrical properties, as well as histomorphometry and immunolocalization of VEGF, TIMP2, MMP13, BMP2 in the bone and cartilage tissue of the humerus of their newborn piglets. PloS One, 16: 2 e024-0642.

Vu J.P., Larauche M., Flores M., Luong L., Norris J., Oh S., Liang L.J., Waschek J., Pisegna J.R., Germano P.M. (2015). Regulation of appetite, body composition, and metabolic hormones by vasoactive intestinal polypeptide (VIP). J. Mol. Neurosci., 56: 377-387.

Winzell M.S., Ahrén B. (2007). Role of VIP and PACAP in islet function. Peptides, 28: 1805-1813.

Xiong X., Tan B., Song M., Ji P., Kim K., Yin Y., Liu Y. (2019). Nutritional intervention for the intestinal development and health of weaned pigs. Front. Vet. Sci., 6: 46.

Yavas C., Yavas G., Acar H., Toy H., Yuce D., Akyurek S., Ata O. (2013). Amelioration of radiation-induced acute inflammation and mucosal atrophy by beta-hydroxy-beta-methylbutyrate, L-glutamine, and L-argınıne: results of an experimental study. Support Care Cancer, 21: 883-888.

Zheng C., Song B., Duan Y., Zhong Y., Yan Z., Zhang S., Li F. (2020). Dietary $\beta$-hydroxy- $\beta$-methylbutyrate improves intestinal function in weaned piglets after lipopolysaccharide challenge. Nutrition, 78: 110839 .

Received: 16 VIII 2021

Accepted: 17 IX 2021 